Annales Geophysicae (2001) 19: 395-409 (C) European Geophysical Society 2001

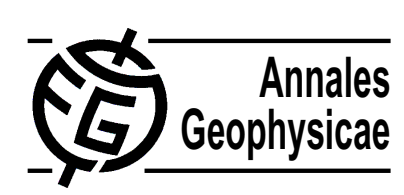

\title{
The shock-acoustic waves generated by earthquakes
}

\author{
E. L. Afraimovich, N. P. Perevalova, A. V. Plotnikov, and A. M. Uralov \\ Institute of Solar-Terrestrial Physics SD RAS, P. O. Box 4026, Irkutsk, 664033, Russia
}

Received: 6 July 2000 - Revised: 5 February 2001 - Accepted: 6 March 2001

\begin{abstract}
We investigate the form and dynamics of shockacoustic waves generated by earthquakes. We use the method for detecting and locating the sources of ionospheric impulsive disturbances, based on using data from a global network of receivers of the GPS navigation system, and require no a priori information about the place and time of the associated effects. The practical implementation of the method is illustrated by a case study of earthquake effects in Turkey (17 August and 12 November 1999), in Southern Sumatra (4 June 2000), and off the coast of Central America (13 January 2001). It was found that in all instances the time period of the ionospheric response is 180-390 s, and the amplitude exceeds, by a factor of two as a minimum, the standard deviation of background fluctuations in total electron content in this range of periods under quiet and moderate geomagnetic conditions. The elevation of the wave vector varies through a range of $20-44^{\circ}$, and the phase velocity (1100$1300 \mathrm{~m} / \mathrm{s}$ ) approaches the sound velocity at the heights of the ionospheric F-region maximum. The calculated (by neglecting refraction corrections) location of the source roughly corresponds to the earthquake epicenter. Our data are consistent with the present views that shock-acoustic waves are caused by a piston-like movement of the Earth's surface in the zone of an earthquake epicenter.
\end{abstract}

Key words. Ionosphere (ionospheric disturbances; wave propagation) - Radio science (ionospheric propagation)

\section{Introduction}

A plethora of publications have been devoted to the study of the ionospheric response to disturbances arising from impulsive forcing on the Earth's atmosphere. It was found that in many cases a high proportion of the energy of the initial atmospheric disturbance is concentrated in the acoustic shock wave. Large earthquakes also provide a natural source of impulsive forcing.

Correspondence to: E. L. Afraimovich (afra@iszf.irk.ru)
These investigations also have important practical implications since they furnish a means of substantiating reliable signal indications of technogenic effects, which is necessary for the construction of an effective global radiophysical system for the detection and localization of these effects. Essentially, existing global systems with such a purpose use different processing techniques for infrasound and seismic signals. However, in connection with the expansion of the geography and the types of technogenic impact on the environment, very challenging problems, heretofore, have been those which improve the sensitivity of detection and the reliability of measured parameters of the sources of impacts, based also on using independent measurements of the entire spectrum of signals generated during such effects.

To solve the above problems requires reliable information about fundamental parameters of the ionospheric response to the shock wave, such as the amplitude and the form, the period, the phase and group velocity of the wavetrain, as well as angular characteristics of the wave vector. Note that for naming the ionospheric response of the shock wave, the literature uses terminology incorporating a different physical interpretation, among them the term 'shock-acoustic wave' (SAW) (Nagorsky, 1998). For convenience in this paper, we shall use this term despite the fact that it does not reflect essentially the physical nature of the phenomenon.

There is a wide scatter of published data on the main parameters of SAWs generated during industrial explosions and earthquakes. The oscillation period of the ionospheric response of SAWs varied from 30 to $300 \mathrm{~s}$, and the propagation velocity fluctuated from 700 to $1200 \mathrm{~m} / \mathrm{s}$ (Nagorsky, 1998; Fitzgerald, 1997; Calais et al., 1998; Afraimovich et al., 1984; Blanc and Jacobson, 1989).

The lack of comprehensive, reliable data on SAW parameters is due primarily to the limitations of existing experimental methods and detection facilities. The main body of data was obtained by measuring the frequency Doppler shift at vertical and oblique-incidence ionospheric soundings in the HF range (Nagorsky, 1998; Afraimovich et al., 1984; Jacobson and Carlos, 1994). In some instances, the sensitivity of 
Table 1. General information about earthquakes

\begin{tabular}{|c|c|c|c|c|c|c|}
\hline $\mathrm{N}$ & Epicenter & Data & $\begin{array}{c}t_{0} \\
(\mathrm{UT})\end{array}$ & $\begin{array}{c}\text { Depth } \\
(\mathrm{km})\end{array}$ & $\begin{array}{c}\text { Magnitude } \\
\text { mb Ms Mw }\end{array}$ & $\begin{array}{c}\text { DST } \\
(\mathrm{nT})\end{array}$ \\
\hline 1 & $40.70 \mathrm{~N}, 29.99 \mathrm{E}$ & 17 Aug 1999 (DAY 229) & $00: 01: 39$ & 17 & 6.37 .87 .4 & -14 \\
\hline 2 & $40.79 \mathrm{~N}, 31.11 \mathrm{E}$ & 12 Nov 1999 (DAY 316) & $16: 57: 20$ & 10 & 6.57 .57 .1 & -44 \\
\hline 3 & $4.72 \mathrm{~S}, 102.1 \mathrm{E}$ & 4 Jun 2000 (DAY 156) & $16: 28: 26$ & 33 & 6.88 .07 .7 & +8 \\
\hline 4 & $12.83 \mathrm{~N}, 88.79 \mathrm{~W}$ & 13 Jan 2001 (DAY 013) & $17: 33: 29$ & 39 & --7.6 & +4 \\
\hline
\end{tabular}

this method is sufficient to detect the SAW reliably; however, difficulties emerge for localizing the region where the detected signal is generated. These problems are caused by the multiple-hop character of HF signal propagation. This provides no chance of deriving reliable information about the phase and group velocities of the SAW propagation, of estimating angular characteristics of the wave vector, and, further still, of localizing the SAW source.

Using the method of transionospheric sounding with VHF radio signals from geostationary satellites, a number of experimental data on SAW parameters were obtained in measurements of the Faraday rotation of the plane of signal polarization, which is proportional to a total electron content (TEC) along the line connecting the satellite-borne transmitter with the receiver ( $\mathrm{Li}$ et al., 1994).

A common drawback of the above-mentioned methods when determining the SAW phase velocity is the necessity of knowing the time of events, since this velocity is inferred from the SAW delay with respect to the time of events, assuming that the velocity is constant along the propagation path, which is quite contrary to fact. For determining the above-mentioned fairly complete set of SAW parameters, it is necessary to have appropriate spatial and temporal resolution, which cannot be ensured by the existing very sparse networks of ionosondes, oblique-incidence radio sounding paths, and incoherent scatter radars.

The advent of the Global Positioning System (GPS), plus the subsequent creation of extensive networks of GPS stations (at least 757 sites as of November 2000), with their data being now available via the Internet, has opened up a new era in remote ionospheric sensing.

Currently, some authors have embarked on an intense development of methods for detecting the ionospheric response of strong earthquakes (Calais and Minster, 1995), rocket launchings (Calais and Minster, 1996), and industrial surface explosions (Fitzgerald, 1997; Calais et al., 1998). In the cited references, the SAW phase velocity was determined by the 'crossing' method, by estimating the time delay of SAW arrival at subionospheric points corresponding to different GPS satellites observed at a given time. However, the accuracy of such a method is rather low because the altitude at which the subionospheric points are specified is determined in a crude way.

The goal of this paper is to describe a method for determining parameters of the SAW generated by earthquakes (including the phase velocity, angular characteristics of the
SAW wave vector, the direction towards the source, and the source location) using GPS-arrays whose elements can be chosen out of a large set of GPS stations from the global GPS network. Section 2 presents a description of the experimental geometry, and general information about the earthquakes under consideration. The proposed method is briefly outlined in Sect. 3. Results of measurements of SAW parameters from different GPS arrays during earthquakes are presented in Sect. 4. Section 5 is devoted to the discussion of experimental results, including analytical simulation results.

\section{The geometry and general characterization of experi- ments}

Detection results on two earthquakes in Turkey (17 August and 12 November 1999), in Southern Sumatra (4 June 2000), and off the coast of Central America (13 January 2001) are presented below. The information about the earthquakes was acquired via the Internet (http://earthquake.usgs.gov). General information about these earthquakes is presented in $\mathrm{Ta}$ ble 1 (including the time of the main shock, $t_{0}$, in the universal time UT, the position of the earthquake epicenter, the depth and the magnitude, as well as the level of geomagnetic disturbance from the data on $D s t$-variations). It was found that the deviation of Dst for the selected days was quite moderate, which enabled the SAWs to be identified.

Figure 1 illustrates the experimental geometry during the earthquakes in Turkey (a), off the coast of Central America (b), and in Southern Sumatra (c).

In spite of the small number of GPS stations in the earthquake area, we were able to use a sufficient number of them for the implementation of the proposed method. Table 2 presents the geographic coordinates of the GPS stations used as GPS array elements.

\section{Methods of determining shock-acoustic wave charac- teristics using GPS-arrays}

The standard GPS technology provides a means for wave disturbances detection based on phase measurements of TEC at each of the spatially separated two-frequency GPS receivers. A method of reconstructing TEC variations was detailed and validated in a series of publications (Calais and Minster, 1995, 1996; Fitzgerald, 1997). We reproduce here only the final 
TURKEY EARTHQUAKES

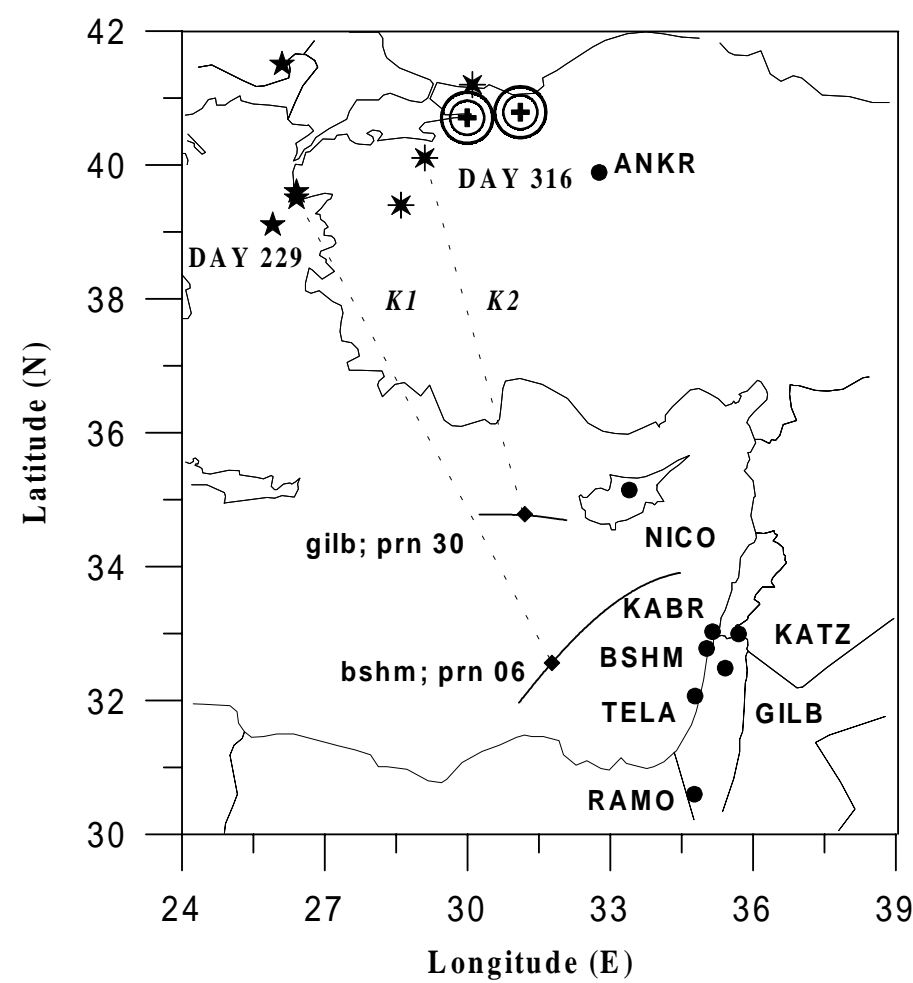

a.

EARTHQUAKE OFF COAST OF CENTRAL AMERICA AND SOUTHERN SUMATRA

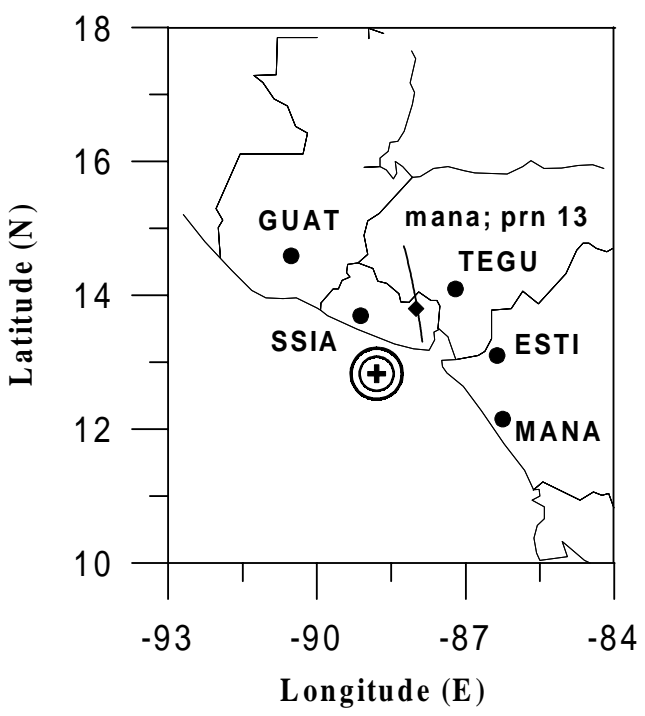

b.

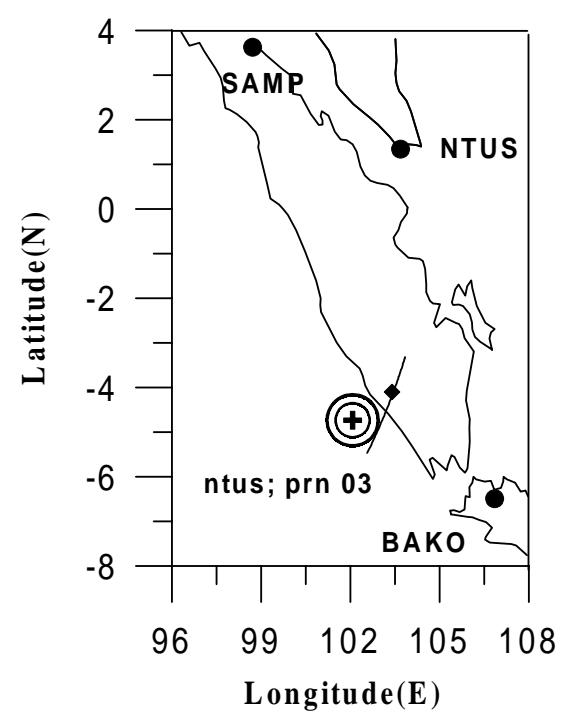

c.

Fig. 1. Experimental geometry during the earthquakes in Turkey - (a). Crosses show the positions of the earthquake epicenters. Solid curves represent the trajectories of the subionospheric points for each GPS satellite at the height $h_{i}=400 \mathrm{~km}$. Dark diamonds along the trajectories correspond to the coordinates of the subionospheric points at time $t_{p}$ of a maximum deviation of the TEC. Heavy dots and large lettering show the location and the names of the GPS stations, while lower-case letters along the trajectories refer to station names and PRN numbers of the GPS satellites for these trajectories. Asterisks mark the source location at $0 \mathrm{~km}$ altitude inferred from the data from the GPS arrays. Numbers at the asterisks correspond to the respective day numbers. Straight dashed lines that connect the expected source and the subionospheric point represent the horizontal projection of the wave vector $\boldsymbol{K}_{t}$. The scaling of the coordinate axes is chosen from considerations of an approximate equality of the linear dimensions along the latitude and longitude. The same, but for the earthquakes off the coast of Central America on 13 January 2001 - (b), and in Southern Sumatra on 4 June 2000 - (c). 
Table 2. GPS-sites and location

\begin{tabular}{|c|c|c|c||c|c|c|c|}
\hline N & Sites & $\begin{array}{c}\text { Geograph. } \\
\text { latitude }\end{array}$ & $\begin{array}{c}\text { Geograph. } \\
\text { longitude }\end{array}$ & $\mathrm{N}$ & Sites & $\begin{array}{c}\text { Geograph. } \\
\text { latitude }\end{array}$ & $\begin{array}{c}\text { Geograph. } \\
\text { longitude }\end{array}$ \\
\hline \hline 1 & ANKR & 39.887 & 32.759 & 9 & TEGU & 14.090 & -87.206 \\
\hline 2 & BSHM & 32.778 & 35.022 & 10 & SSIA & 13.697 & -89.117 \\
\hline 3 & GILB & 32.479 & 35.416 & 11 & MANA & 12.149 & -86.2489 \\
\hline 4 & KABR & 33.022 & 35.145 & 12 & GUAT & 14.590 & -90.520 \\
\hline 5 & KATZ & 32.995 & 35.688 & 13 & ESTI & 13.100 & -86.3621 \\
\hline 6 & NICO & 35.141 & 33.396 & 14 & SAMP & 3.622 & 98.715 \\
\hline 7 & RAMO & 30.597 & 34.763 & 15 & NTUS & 1.346 & 103.680 \\
\hline 8 & TELA & 32.067 & 34.780 & 16 & BAKO & -6.491 & 106.849 \\
\hline
\end{tabular}

formula for the total electron content (I)

$$
I=\frac{1}{40.308} \frac{f_{1}^{2} f_{2}^{2}}{f_{1}^{2}-f_{2}^{2}}\left[\left(L_{1} \lambda_{1}-L_{2} \lambda_{2}\right)+\text { const }+n L\right]
$$

where $L_{1} \lambda_{1}$ and $L_{2} \lambda_{2}$ are additional paths of the radio signal caused by the phase delay in the ionosphere, (in m); $L_{1}$ and $L_{2}$ represent the number of phase rotations at the frequencies $f_{1}$ and $f_{2} ; \lambda_{1}$ and $\lambda_{2}$ stand for the corresponding wavelengths, (in $\mathrm{m}$ ); const is the unknown initial phase ambiguity, (in $\mathrm{m}$ ); and $n L$ are errors in determining the phase path, (in m).

Phase measurements in the GPS can be made with a high degree of accuracy corresponding to the error of TEC determination of at least $10^{14} \mathrm{~m}^{-2}$ when averaged on a 30 -second interval, with some uncertainty of the initial value of TEC, however. This makes possible the detection of ionization irregularities and wave processes in the ionosphere over a wide range of amplitudes (up to $10^{-4}$ of the diurnal TEC variation) and periods (from 24 hours to $5 \mathrm{~min}$ ). The unit of TEC, TECU, is equal to $10^{16} \mathrm{~m}^{-2}$; it is commonly accepted in the literature, and will be used hereafter.

A convenient way of determining the ionospheric response delay of the shock wave involves the frequency Doppler shift $F$ from TEC series, obtained by formula (1). Such an approach is also useful in comparing TEC response characteristics from the GPS data with those obtained by analyzing VHF signals from geostationary satellites, as well as in detecting the shock wave in the HF range. For an approximation sufficient for the purpose of our investigation, a corresponding relationship was obtained by Davies (1969)

$$
F=13.5 \cdot 10^{-8} I_{t}^{\prime} / f
$$

where $I_{t}^{\prime}$ stands for the time derivative of TEC. Relevant results derived from analyzing the $F(t)$ variations calculated for the 'reduced' frequency of $136 \mathrm{MHz}$ are discussed in Sect. 4.

The correspondence of space-time phase characteristics, obtained through transionospheric soundings, with local characteristics of disturbances in the ionosphere, was considered in detail in a wide variety of publications (Afraimovich et al., 1992; Mercier and Jacobson, 1997) and is not analyzed at length in this study. The most important conclusion of the cited references is the fact that, the extensively exploited model of a 'plane phase screen' disturbance $\Delta I(x, y, t)$ of TEC, faithfully copies the horizontal part of the corresponding disturbance of local electron concentration $\Delta N(x, y, z, t)$, independent of the angular position of the source, and thus, can be used in experiments for measuring the TEC disturbances.

However, the TEC response amplitude experiences a strong azimuthal dependence caused by the integral character of a transionospheric sounding. As a first approximation, the transionospheric sounding method is responsive only to Traveling Ionospheric Disturbances (TIDs), with the wave vector $\boldsymbol{K}_{t}$ perpendicular to the direction $\boldsymbol{r}$, which is along the Line-of-Sight (LOS) from the receiver to the satellite. A corresponding condition for elevation $\theta$ and azimuth $\alpha$ of an arbitrary wave vector $\boldsymbol{K}_{t}$ normal to the direction $\boldsymbol{r}$, has the form

$\theta=\arctan \left(-\cos \left(\alpha_{s}-\alpha\right) / \tan \theta_{s}\right)$

where $\alpha_{s}$ is the azimuthal angle measured east to north, and $\theta_{S}$ is the angle of elevation of the satellite at the receiver.

We used formula (3) to determine the elevation $\theta$ of $\boldsymbol{K}_{t}$ from the known mean value of azimuth $\alpha$ by Afraimovich et al. (1998) - see Sects. 3.2 and 4.

3.1 Detection and determination of the horizontal phase velocity and the direction of the SAW phase front along the ground by GPS-arrays

In the simplest form, space-time variations of the TEC $\Delta I(t, x, y)$ in the ionosphere, at each given time $t$, can be represented in terms of the phase interference pattern that moves without a change in its shape (the solitary, plane travelling wave)

$\Delta I(t, x, y)=\delta \sin \left(\Omega t-K_{x} x-K_{y} y+\varphi_{0}\right)$

where $\delta, K_{x}, K_{y}, \Omega$, are the amplitude, the $x$ - and $y$-projections of the wave vector $\boldsymbol{K}$, and the angular frequency of the disturbance, respectively; $T=2 \pi / \Omega, \Lambda=2 \pi /|\boldsymbol{K}|$ is its period and wavelength; and $\varphi_{0}$ is the initial phase of the 
disturbance. The vector $\boldsymbol{K}$ is a horizontal projection of the full vector $\boldsymbol{K}_{t}$.

At this point, it is assumed that in the case of small spatial and temporal increments (the distances between GPS-array sites are less than the typical spatial scale $\Lambda$ of TEC variation, and the time interval between counts is less than the corresponding time scale), the influence of second derivatives can be neglected. The following choices of GPS-arrays meet these requirements.

We now summarize briefly the sequence of data processing procedures. Out of a large number of GPS stations, three sites $(\mathrm{A}, \mathrm{B}, \mathrm{C})$ are selected within distances not exceeding about one-half of the expected wavelength $\Lambda$ of the perturbation. Site B is taken to be the center of a topocentric reference frame whose axis $x$ is directed east, and whose axis $y$ is directed north. The receivers in this frame of reference have the coordinates $\left(x_{A}, y_{A}\right),(0,0),\left(x_{C}, y_{C}\right)$; such a configuration of the GPS receivers represents the GPS-array with a minimum number of the required elements. In regions with a dense network of GPS sites, we can obtain a large variety of GPS-arrays of a different configuration, enabling the acquired data to be checked for reliability; in this paper, we have exploited this possibility.

The input data includes series of slant TEC values $I_{A}(t)$, $I_{B}(t), I(t)$, as well as the corresponding series of elevation values $\theta_{S}(t)$, and the azimuth $\alpha_{s}(t)$ of the LOS. For determining SAW characteristics, continuous series of measurements of $I_{A}(t), I_{B}(t), I_{C}(t)$ are selected with a length of at least a one-hour interval, which includes the time of a earthquake.

To eliminate spatio-temporal variations of the regular ionosphere, as well as trends introduced by the orbital motion of the satellite, a procedure involving a preliminary smoothing of the initial series with the selected time window is used to remove the trend. This procedure is better suited to the detection of a single pulse signal ( $N$-wave) than the frequently used band-pass filter ( $\mathrm{Li}$ et al., 1994; Calais and Minster, 1995, 1996; Fitzgerald, 1997; Calais et al., 1998). A limitation of the band-pass filter is the oscillatory character of the response which prevents it from reconstructing the form of the $N$-wave.

Elevation $\theta_{s}(t)$ and azimuth $\alpha_{S}(t)$ values of the LOS are used to determine the location of the subionospheric point, as well as to calculate the elevation $\theta$ of the wave vector $\boldsymbol{K}_{t}$ of the disturbance from the known azimuth $\alpha$ (see formula (3)).

The most reliable results from the determination of SAW parameters correspond to high values of elevations $\theta_{s}(t)$ of the LOS because sphericity effects become reasonably small. In addition, there is no need to convert the slant TEC $\Delta I(t)$ to a 'vertical' value. In this paper, all results were obtained for elevations $\theta_{s}(t)$ larger than $30^{\circ}$.

Since the distance between GPS-array elements (from several tens of kilometers to a few hundred kilometers) is much smaller than the distance to the GPS satellite (over $20000 \mathrm{~km}$ ), the array geometry at the height of the ionosphere is identical to that on the ground.

Figure 2a shows typical time dependencies of a slant TEC $I(t)$ at the GPS-array BSHM station near the area of the earthquake of 17 August 1999 (heavy curve), one day before and after the earthquake (thin lines). For the same days, panel b shows TEC variations $\Delta I(t)$ after removal of a linear trend and smoothing by averaging over a sliding window of 5 min. Variations in frequency Doppler shift $F(t)$, 'reduced' to the sounding signal frequency of $136 \mathrm{MHz}$ for three sites of the array (KATZ BSHM GILB) on 17 August 1999, are presented in panel $\mathrm{c}$.

Figure 2 shows that fast $N$-shaped oscillations, with a typical period of about $390 \mathrm{~s}$, are distinguished among slow TEC variations. The oscillation amplitude (up to 0.12 TECU) is far in excess of the background TEC fluctuation intensity, as seen on the days before and after the earthquake. Variations in frequency Doppler shift $F(t)$ for spatially separated sites (KATZ BSHM GILB) are well correlated but are shifted relative to each other by an amount well below the period, which permits the SAW propagation velocity to be unambiguously determined. The $30 \mathrm{~s}$ sampling rate of the GPS data is not quite sufficient for determining small shifts of such signals with an adequate accuracy for different sites of the array. Therefore, we used a parabolic approximation of the $F(t)$ oscillations in the neighborhood of minimum $F(t)$, which is quite acceptable when the signal/noise ratio is high.

Taking into account the good signal/noise ratio (better than 1), and knowing the coordinates of the array sites A, B and C, we determine the horizontal projection of the phase velocity $V_{h}$ from time shifts $t_{p}$ of a maximum deviation of the frequency Doppler shift $F(t)$. Preliminarily measured shifts are subjected to a linear transformation with the purpose of calculating shifts for sites spaced relative to the central site northward $N$ and eastward $E$. This is followed by a calculation of the $E$ - and $N$-components of $V_{x}$ and $V_{y}$, as well as the direction $\alpha$ in the range of angles $0-360^{\circ}$ and the modulus $V_{h}$ of the horizontal component of the SAW phase velocity

$\alpha=\arctan \left(V_{y} / V_{x}\right)$

$V_{h}=\left|V_{x} V_{y}\right|\left(V_{x}^{2}+V_{y}^{2}\right)^{-1 / 2}$

where $V_{y}, V_{x}$ are the velocities with which the phase front crosses the axes $x$ and $y$. The orientation $\alpha$ of the wave vector $\boldsymbol{K}$, which is coincident with the propagation azimuth of the SAW phase front, is calculated unambiguously in the range $0-360^{\circ}$, subject to the condition that $\arctan \left(V_{y} / V_{x}\right)$ is calculated with regard to the sign of the numerator and denominator.

The above method for determining the SAW phase velocity neglects the correction for orbital motion of the satellite because the estimates of $V_{h}$, obtained below, exceed an order of magnitude, as a minimum, of the velocity of the subionospheric point at the height of the ionosphere for elevations $\theta_{s}>30^{\circ}$ (Afraimovich et al., 1998).

Obviously, the method presented above can be used if the distance between GPS stations is much shorter than the TEC disturbance wavelength $\Lambda$, and the distance from the earthquake epicenter to the array. This corresponds to the detection condition in the far-field zone. 


\section{TURKEY EARTHQUAKES}

\subsubsection{9}
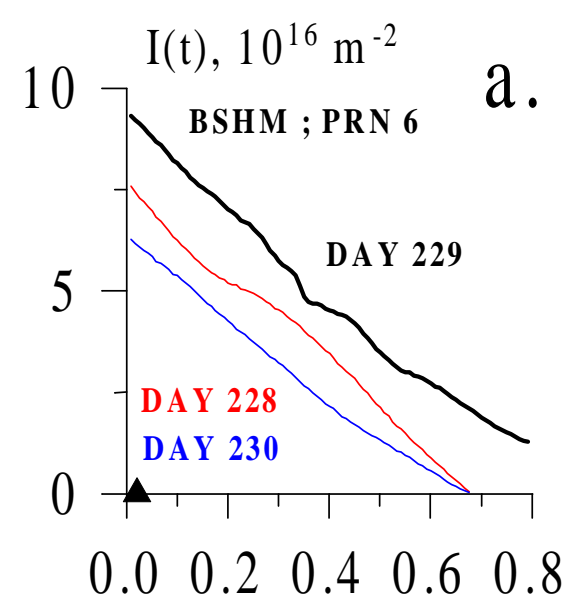

$\Delta \mathrm{I}(\mathrm{t}), 10^{16} \mathrm{~m}^{-2}$

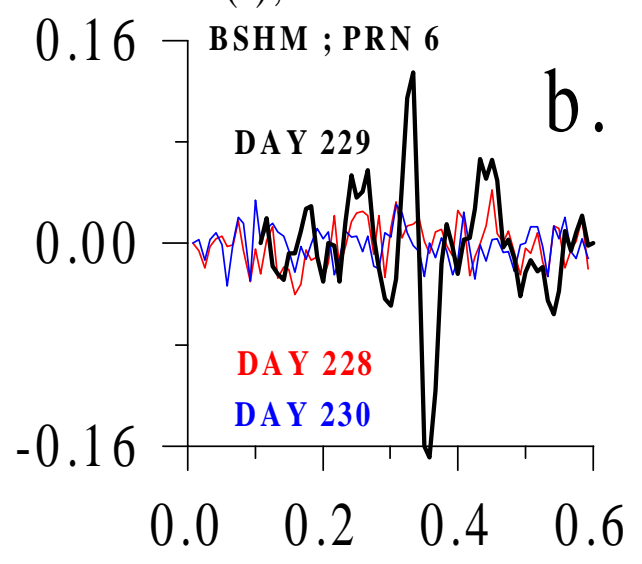

$\mathrm{F}(\mathrm{t}), \mathrm{Hz}$

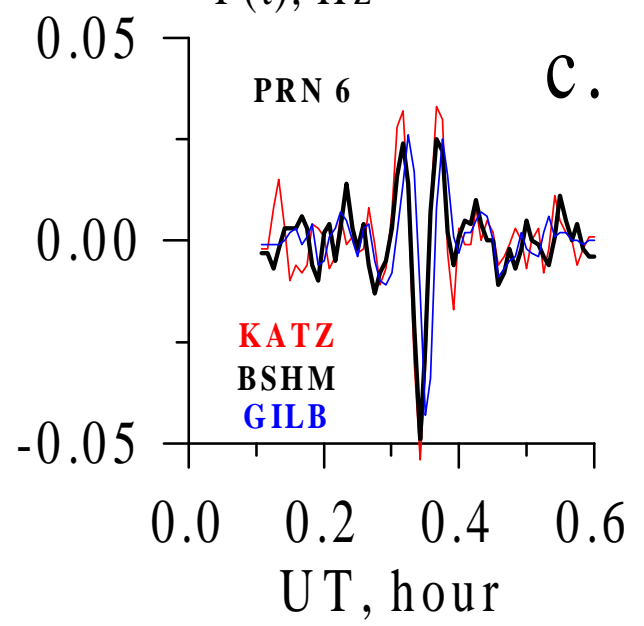

12.11.1999
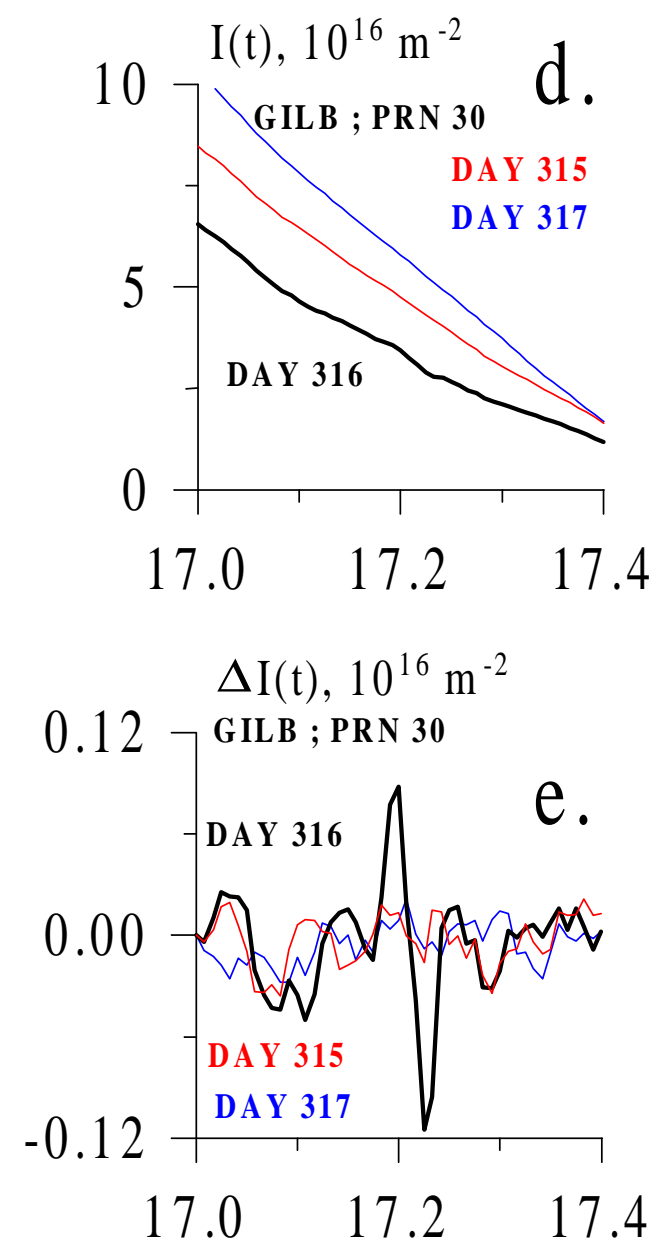

$\mathrm{F}(\mathrm{t}), \mathrm{Hz}$

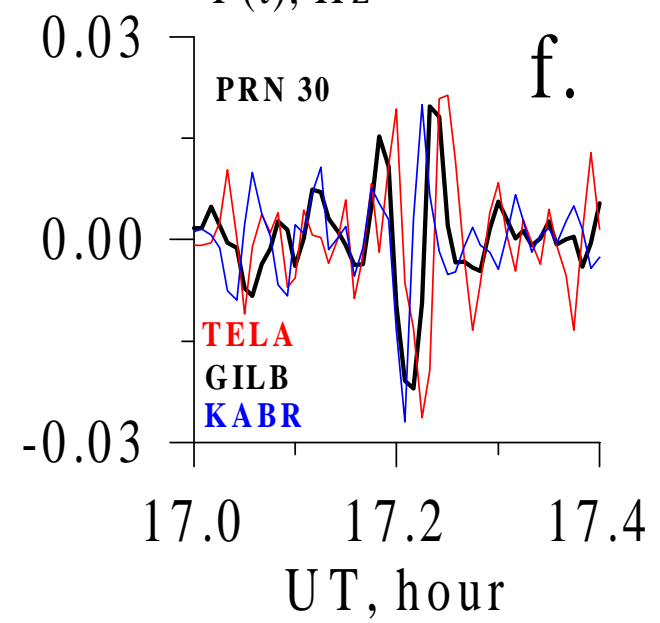

Fig. 2. Time dependencies of slant TEC $I(t)$ at one of the three sites of the GPS-array in the area of the earthquakes on 17 August and 12 November 1999, on the day of earthquake (heavy curve), and one day before and one day after the event (thin curves) - panels (a,d). Panels $(\mathbf{b}, \mathbf{e})$ - for the same days but the TEC variations $\Delta I(t)$ with the linear trend removed and with a smoothing with a time window of 5 min; panels (c,f) - variations of the frequency Doppler shift $F(t)$ 'reduced' to the sounding signal frequency of $136 \mathrm{MHz}$, for three sites of the arrays, days of earthquake. All panels show day numbers, GPS station names, and PRN numbers of the GPS satellites. 
From the delay $\Delta t=t_{p}-t_{0}$ and the known path length between the earthquake focus and the subionospheric point, we also calculated the SAW mean velocity $V_{a}$, in order to compare our estimates of the SAW phase velocity with the usually used method of measuring this quantity.

3.2 Determination of the wave vector elevation $\theta$ and the velocity modulus $V_{t}$ of the shock wave

Afraimovich et al. (1992) showed that for the Gaussian ionization distribution, the TEC disturbance amplitude $(M)$ is determined by the aspect angle $\gamma$ between the vectors $\boldsymbol{K}_{t}$ and $\boldsymbol{r}$, as well as by the ratio of the wavelength of the disturbance $\Lambda$ to the half-thickness of the ionization maximum $h_{d}$

$M \propto \exp \left(-\frac{\pi^{2} h_{d}^{2} \cos ^{2} \gamma}{\Lambda^{2} \cos ^{2} \theta_{s}}\right)$.

In the case under consideration (see below), for a phase velocity on the order of $1 \mathrm{~km} / \mathrm{s}$ and for a period of about $200 \mathrm{~s}$, the wavelength $\Lambda$ is comparable with the half-thickness of the ionization maximum $h_{d}$. When the elevations $\theta_{s}$ are $30^{\circ}$, $45^{\circ}, 60^{\circ}$, the 'beam-width' $M(\gamma)$, at the 0.5 level, is $25^{\circ}$, $22^{\circ}$ and $15^{\circ}$, respectively. If $h_{d}$ is twice as large as the wavelength, then the beam tapers to $14^{\circ}, 10^{\circ}$ and $8^{\circ}$, respectively.

The beam-width is sufficiently small that the aspect condition (3) restricts the number of beam trajectories to the satellite, for which it is possible to detect, with reliably, the SAW response in the presence of noise (near the angles $\gamma=90^{\circ}$ ). On the other hand, formula (3) can be used to determine the elevation $\theta$ of the wave vector $\boldsymbol{K}_{t}$ of the shock wave at the known value of the azimuth $\alpha$ (Afraimovich et al., 1998). Hence, the phase velocity modulus $V_{t}$ can be defined as

$V_{t}=V_{h} \cos (\theta)$

The above values of the width $M(\gamma)$ determine the error of calculation of the elevations $\theta$ (of the order of $20^{\circ}$ to the above conditions).

3.3 Determining the position of the SAW source without regard for refraction corrections

The ionospheric region that is responsible for the main contribution to TEC variations lies in the neighborhood of the maximum of the ionospheric $F$-region, which does determine the height $h_{i}$ of the penetration point. When selecting $h_{i}$, it should be taken into consideration that the decrease in electron density, with height above the main maximum of the F2-layer, proceeds much slower than below this maximum. Since the density distribution with height is essentially a 'weight function' of the TEC response to a wave disturbance (Afraimovich et al., 1992), it is appropriate to use, as $h_{i}$, the value exceeding the true height of the layer $h_{m} \mathrm{~F} 2$ maximum by about $100 \mathrm{~km} . h_{m} \mathrm{~F} 2$ varies between 250 and $350 \mathrm{~km}$ depending on the time of day and on some geophysical factors which, when necessary, can be taken into account if additional experimental data and current ionospheric models are available. In all calculations that follow, $h_{i}=400 \mathrm{~km}$ is used.

To a first approximation, it can be assumed that the imaginary detector, which records the ionospheric SAW response in TEC variations, is located at this altitude. The horizontal extent of the detection region, which can be inferred from the propagation velocity of the subionospheric point as a consequence of the orbital motion of the GPS satellite (on the order of 70-150 m/s; see Pi at al., 1997), and from the SAW period (on the order of $200 \mathrm{~s}$; see Sect. 4), does not exceed 20-40 km, which is far smaller than its 'vertical size' (on the order of the half-thickness of the ionization maximum $h_{d}$ ).

From the GPS data, we can determine the coordinates $X_{s}$ and $Y_{S}$ of the subionospheric point in the horizontal plane $X 0 Y$ of a topocentric frame of reference centered on the point $B(0,0)$ at the time of a maximum TEC deviation, caused by the arrival of the SAW at this point. Since we know the angular coordinates $\theta$ and $\alpha$ of the wave vector $\boldsymbol{K}_{t}$, it is possible to determine the location of the point at which this vector intersects the horizontal plane $X^{\prime} 0 Y^{\prime}$ at the height $h_{w}$ of the assumed source. Assuming a rectilinear propagation of the SAW from the source to the subionospheric point and neglecting the Earth sphericity, the coordinates $X_{w}$ and $Y_{w}$ of the source in a topocentric frame of reference can be defined as

$$
\begin{aligned}
& X_{w}=X_{p}-\left(h_{i}-h_{w}\right) \frac{\cos \theta \sin \alpha}{\sin \theta} \\
& Y_{w}=Y_{p}-\left(h_{i}-h_{w}\right) \frac{\cos \theta \cos \alpha}{\sin \theta}
\end{aligned}
$$

The coordinates $X_{w}$ and $Y_{w}$, thus obtained, can readily be recalculated to the values of the latitude and longitude $\left(\phi_{w}\right.$ and $\lambda_{w}$ ) of the source. For SAW generated during earthquakes, industrial explosions and underground tests of nuclear devices, $h_{w}$ is taken to be equal to 0 (the source lying at the ground level).

\section{Results of measurements}

Hence, using the transformations described in Sect. 3, we obtain the parameters set determined from TEC variations and characterizing the SAW (see Table 3).

Let us consider the results derived from analyzing the ionospheric effect of SAW during earthquake 17 August 1999, obtained at the array (KATZ, BSHM, GILB) for PRN 6 (at the left of Fig. 2, and line 2 in Table 3).

In this case, the delay of the SAW response, with respect to the time of the earthquake, is $20 \mathrm{~min}$ (DAY 229). The SAW has the form of an $N$-wave with a period $T$ of about $390 \mathrm{~s}$ and an amplitude $A_{I}=0.12$ TECU, which is an order of magnitude larger than TEC fluctuations for background days (DAY 228, DAY 230). It should be noted, however, that this time interval was characterized by a very low level of geomagnetic activity $(-14 \mathrm{nT})$. 
Table 3. The parameters of shock-acoustic waves

\begin{tabular}{|c|c|c|c|c|c|c|c|c|c|c|c|c|}
\hline No. & Sites & $\begin{array}{c}t_{p} \\
\text { (UT) }\end{array}$ & $\begin{array}{c}\Delta t, \\
\text { sec. }\end{array}$ & $\begin{array}{r}T, \\
\text { sec. }\end{array}$ & $\begin{array}{r}A_{I}, \\
\text { TECU }\end{array}$ & $\begin{array}{l}A_{F}, \\
\mathrm{~Hz}\end{array}$ & $\theta,^{\circ}$ & $\alpha,{ }^{\circ}$ & $\begin{array}{c}V_{h}, \\
\mathrm{~m} / \mathrm{s}\end{array}$ & $\begin{array}{c}V_{t}, \\
\mathrm{~m} / \mathrm{s}\end{array}$ & $\begin{array}{c}V_{\alpha}, \\
\mathrm{m} / \mathrm{s}\end{array}$ & $\begin{array}{l}\varphi_{w},{ }^{\circ} \\
\lambda_{w},{ }^{\circ}\end{array}$ \\
\hline \multicolumn{13}{|c|}{17 Aug 1999; $t_{0}=00: 01: 39 \mathrm{UT}$} \\
\hline 1 & $\begin{array}{l}\text { KABR } \\
\text { BSHM } \\
\text { KATZ }\end{array}$ & $\begin{array}{l}00: 21: 04 \\
00: 21: 19\end{array}$ & $\begin{array}{l}1165 \\
1180\end{array}$ & $\begin{array}{l}360 \\
300\end{array}$ & $\begin{array}{l}0.15 \\
0.14\end{array}$ & $\begin{array}{l}0.037 \\
0.049\end{array}$ & 24.9 & 155 & 1296 & 1174 & $\begin{array}{l}873 \\
862\end{array}$ & $\begin{array}{l}39.6 \\
26.4\end{array}$ \\
\hline 2 & $\begin{array}{l}\text { GILB } \\
\text { KATZ } \\
\text { BSHM }\end{array}$ & $00: 21: 54$ & 1215 & 390 & 0.12 & 0.043 & 26.1 & 154 & 1307 & 1174 & 868 & $\begin{array}{l}39.1 \\
25.9\end{array}$ \\
\hline 3 & $\begin{array}{l}\text { KATZ } \\
\text { KABR } \\
\text { GILB }\end{array}$ & $00: 21: 23$ & 1184 & 360 & 0.19 & 0.05 & 25.1 & 155 & 1303 & 1179 & 854 & $\begin{array}{l}39.5 \\
26.4\end{array}$ \\
\hline 4 & $\begin{array}{l}\text { TELA } \\
\text { BSHM } \\
\text { GILB }\end{array}$ & $00: 22: 14$ & 1235 & 360 & 0.1 & 0.026 & 19.9 & 161 & 1238 & 1164 & 894 & $\begin{array}{l}41.5 \\
26.1\end{array}$ \\
\hline 5 & $\sum$ & & & 354 & 0.14 & 0.04 & 24.1 & 156 & 1286 & 1173 & 870 & $\begin{array}{l}39.9 \\
26.2\end{array}$ \\
\hline \multicolumn{13}{|c|}{12 Nov $1999 ; t_{0}=16: 57: 20$ UT } \\
\hline 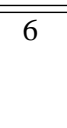 & $\begin{array}{l}\text { KABR } \\
\text { BSHM } \\
\text { GILB }\end{array}$ & $\begin{array}{l}17: 12: 58 \\
17: 13: 21\end{array}$ & $\begin{array}{l}938 \\
961\end{array}$ & $\begin{array}{l}180 \\
180\end{array}$ & $\begin{array}{l}0.06 \\
0.07\end{array}$ & $\begin{array}{l}0.027 \\
0.021\end{array}$ & 29.5 & 194 & 1285 & 1119 & $\begin{array}{l}807 \\
787\end{array}$ & $\begin{array}{l}42.2 \\
30.1\end{array}$ \\
\hline 7 & $\begin{array}{l}\text { TELA } \\
\text { KABR } \\
\text { GILB }\end{array}$ & $17: 14: 09$ & 1009 & 210 & 0.097 & 0.021 & 43.8 & 180 & 1487 & 1073 & 838 & $\begin{array}{l}39.4 \\
28.6\end{array}$ \\
\hline 8 & $\begin{array}{l}\text { GILB } \\
\text { BSHM } \\
\text { TELA }\end{array}$ & $17: 13: 39$ & 979 & 210 & 0.09 & 0.022 & 39.6 & 184 & 1663 & 1280 & 817 & $\begin{array}{l}40.1 \\
29.1\end{array}$ \\
\hline 9 & $\sum$ & & & 195 & 0.079 & 0.023 & 37.6 & 186 & 1478 & 1157 & 812 & $\begin{array}{l}40.5 \\
29.2\end{array}$ \\
\hline \multicolumn{13}{|c|}{ 4 Jun $2000 ; t_{0}=16: 28: 26$ UT } \\
\hline 10 & $\begin{array}{l}\text { SAMP } \\
\text { NTUS } \\
\text { BAKO }\end{array}$ & $\begin{array}{l}16: 46: 12 \\
16: 42: 16 \\
16: 49: 16\end{array}$ & $\begin{array}{l}1092 \\
856 \\
1276\end{array}$ & $\begin{array}{l}270 \\
270 \\
240\end{array}$ & $\begin{array}{l}0.1 \\
0.5 \\
0.06\end{array}$ & $\begin{array}{l}0.04 \\
0.09 \\
0.02\end{array}$ & & & & & $\begin{array}{l}558 \\
503 \\
642\end{array}$ & \\
\hline \multicolumn{13}{|c|}{13 Jan $2001 ; t_{0}=17: 33: 29$ UT } \\
\hline 111 & $\begin{array}{l}\text { TEGU } \\
\text { ESTI } \\
\text { MANA }\end{array}$ & $\begin{array}{l}17: 45: 11 \\
17: 46: 30 \\
17: 47: 21\end{array}$ & $\begin{array}{l}851 \\
930 \\
981\end{array}$ & $\begin{array}{l}240 \\
210 \\
270\end{array}$ & $\begin{array}{l}0.09 \\
0.09 \\
0.2\end{array}$ & $\begin{array}{l}0.03 \\
0.03 \\
0.05\end{array}$ & & & & & $\begin{array}{l}591 \\
488 \\
440\end{array}$ & \\
\hline
\end{tabular}

The amplitude of a maximum frequency Doppler shift $A_{F}$, at the 'reduced' frequency of $136 \mathrm{MHz}$, was found to be 0.04 $\mathrm{Hz}$. In view of the fact that the shift $F$ is inversely proportional to the sounding frequency squared (Davies, 1969), this corresponds to a Doppler shift at the working frequency of $13.6 \mathrm{MHz}$ and the equivalent oblique-incidence sounding path of about $A_{F}=4 \mathrm{~Hz}$.

Solid curves in Fig. 1a represent the trajectories of the subionospheric points for each GPS satellite at the height $h_{i}=400 \mathrm{~km}$ during the time interval 0.0-0.8 UT for $17 \mathrm{Au}-$ gust 1999, and 17.0-17.4 UT for 12 November 1999. Dark diamonds along the trajectories correspond to the coordinates of the subionospheric points at time $t_{p}$ of a maximum devi- ation of the TEC (Fig. 2b,e). Crosses show the positions of the earthquake epicenters. Asterisks mark the source location at $0 \mathrm{~km}$ altitude inferred from the data from the GPS arrays. Numbers at the asterisks correspond to the respective day numbers. Straight dashed lines that connect the expected source and the subionospheric point represent the horizontal projection of the wave vector $\boldsymbol{K}_{t}$.

The azimuth $\alpha$ and elevation $\theta$ of the wave vector $\boldsymbol{K}_{t}$, whose horizontal projection is shown in Fig. 1a by a dashed line and is marked by $K_{1}$, are $154^{\circ}$ and $26^{\circ}$, respectively. The horizontal component and the modulus of the phase velocity were found to be $V_{h}=1307 \mathrm{~m} / \mathrm{s}$ and $V_{t}=1174 \mathrm{~m} / \mathrm{s}$. The source coordinates at $0 \mathrm{~km}$ altitude were determined as 


\section{EARTHQUAKES OFF COAST OF CENTRAL AMERICA AND SOUTHERN SUMATRA}

13.01.2001

$\mathrm{I}(\mathrm{t}), 10^{16} \mathrm{~m}^{-2}$

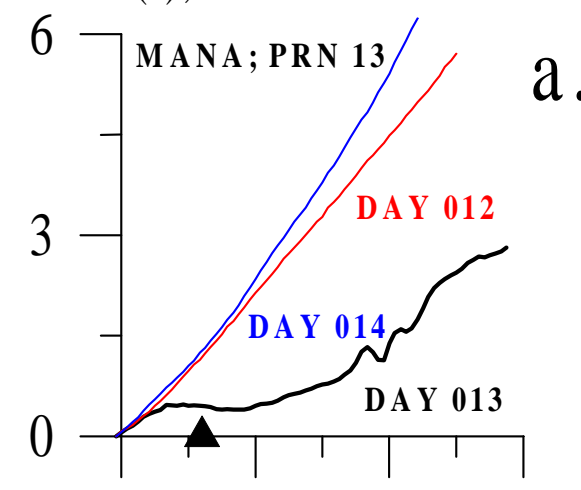

$17.4 \quad 17.6 \quad 17.8 \quad 18.0$

$\Delta \mathrm{I}(\mathrm{t}), 10^{16} \mathrm{~m}^{-2}$

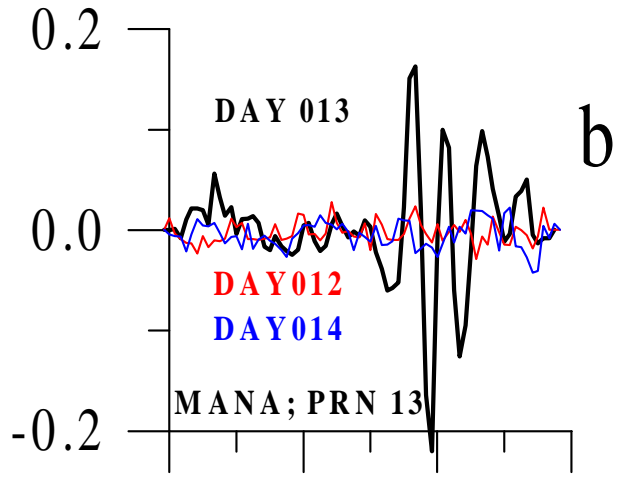

$17.4 \quad 17.6 \quad 17.8 \quad 18.0$

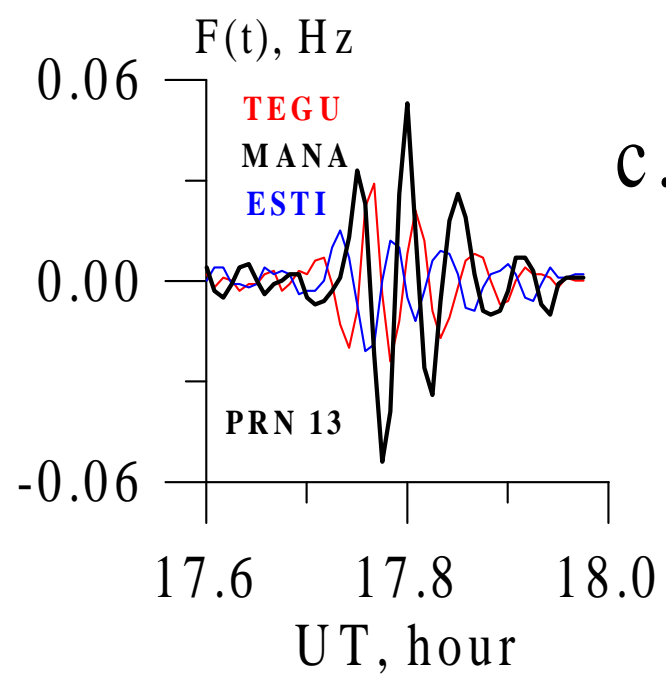

04.06.2000

$\mathrm{I}(\mathrm{t}), 10^{16} \mathrm{~m}^{-2}$

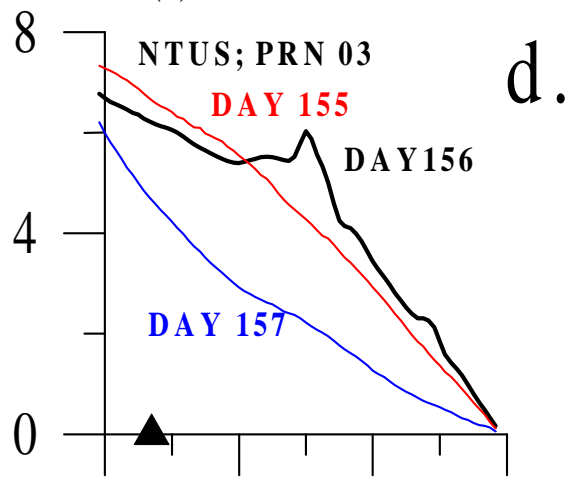

$16.4 \quad 16.6 \quad 16.8 \quad 17.0$

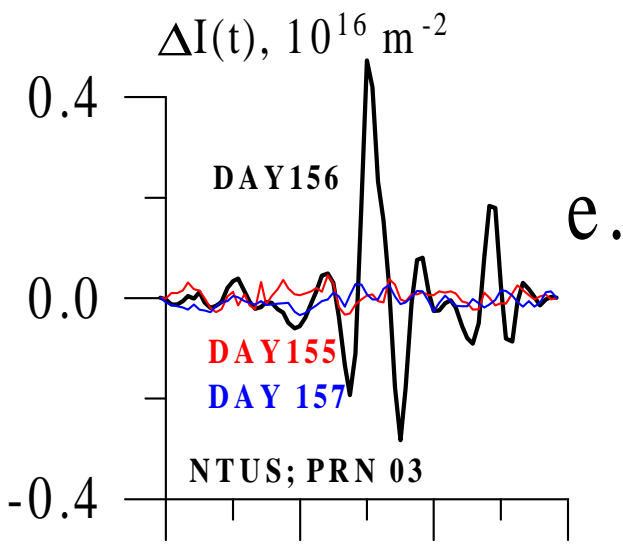

$16.4 \quad 16.6 \quad 16.8 \quad 17.0$

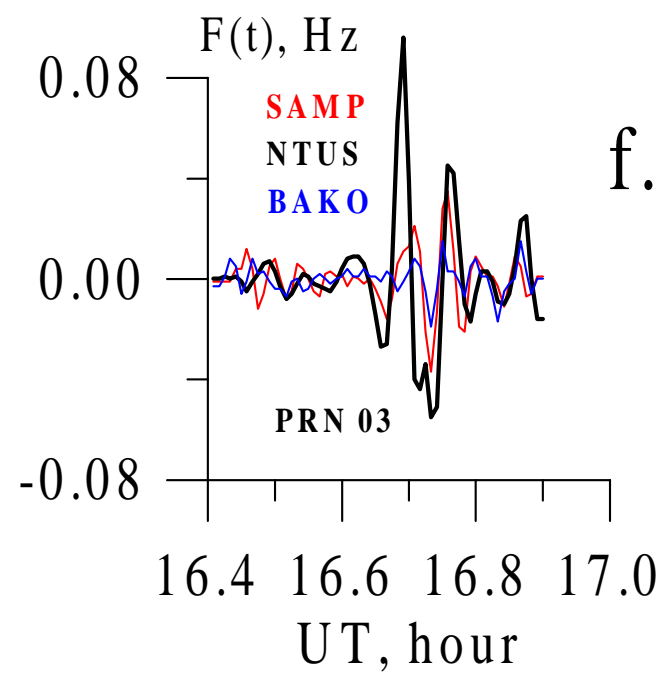

Fig. 3. Same as in Fig. 2, but for the earthquakes off the coast of Central America, 13 January 2001 - at the left, and in Southern Sumatra, 4 June 2000 - at the right. 
$\phi_{w}=39.1^{\circ}$ and $\lambda_{w}=25.9^{\circ}$. The alculated (by neglecting refraction corrections) location of the source was roughly close the earthquake epicenter.

The 'mean' velocity of about $V_{a}=870 \mathrm{~m} / \mathrm{s}$, determined in a usual manner from the response delay with respect to the start, was smaller than the phase velocity $V_{t}$. Conceivably, this is associated with an added delay in the response, as a consequence of the refraction distortions of the SAW path along the LOS, which were neglected in this study.

Similar results for the array (KABR, TELA, GILB) and PRN 30 were also obtained for the earthquake of 12 November 1999. They correspond to the projection of the vector $\boldsymbol{K}_{2}$ in Fig. 1a, the time dependencies in Fig. 2 at the right, and line 7 in Table 3 . The only point worth mentioning here is that the SAW amplitude was somewhat smaller than that of the earthquake on 17 August 1999. With an increased level of geomagnetic activity ( $\sim 44 \mathrm{nT}$ ), this led to a smaller (compared with 17 August 1999) signal/noise ratio; yet this did not preclude reliable estimates of the SAW parameters.

A comparison of the data for both earthquakes showed a reasonably close agreement of SAW parameters, irrespective of the level of geomagnetic disturbance, the season, and the local time.

To convince ourselves that the determination of the main parameters of the SAW form and dynamics is reliable for the earthquakes analyzed here, in the area of the earthquake, we selected different combinations of three sites out of the sets of GPS stations available to us, and these data were processed with the same processing parameters. Relevant results (including the average results for the sets $\Sigma$ ), presented in Table 3 and in Fig. 1a (SAW source position), show that the values of SAW parameters are similar, which indicates a good stability of the data, irrespective of the GPS-array configuration.

The aspect condition (3), corresponding to a maximum amplitude of the TEC response to the transmission of SAWs, was satisfied quite well for this geometry, and simultaneously for all stations. This is confirmed by a high degree of correlation of the SAW responses at the array elements (Fig. 2c,f), which made it possible to obtain different sets of triangles out of the six GPS stations available to us.

The relative position of the GPS stations was highly convenient for determining the SAW parameters during the earthquakes in Turkey, and met the implementation conditions for the method described in Sect. 3.1. Thus, the distance between stations (100-150 km, at most) did not exceed the SAW wavelength of about $200-300 \mathrm{~km}$, and was far less than the distance from the epicenter to the array $(1000 \mathrm{~km})$.

Let us consider the results derived from analyzing the ionospheric effect of SAW during the earthquake on 13 January 2001, obtained at the array (TEGU, MANA, ESTI) for PRN13 (at the left of Fig. 3, and line 11 in Table 3). In this case, the delay of the SAW response, with respect to the time of the earthquake, is 15 min (DAY 013). The SAW has the form of oscillations with a period $T$ of about $270 \mathrm{~s}$, and an amplitude $A_{I}=0.2$ TECU, which is an order of magnitude larger than TEC fluctuations for background days (DAY
012, DAY 014). It should be noted that this time interval was characterized by a very low level of geomagnetic activity (4 nT). Similar results for the array (SAMP, NTUS, BAKO) and PRN 03 were also obtained for the earthquake on 4 June 2000 (at the right of Fig. 3 and line 10 in Table 3). Note that in this case, the response amplitude exceeded twice, as a minimum, that for the other events under consideration. It is not improbable that this is due to the maximum magnitude of the earthquake in Southern Sumatra (see Table 1).

Unfortunately, because of the inadequately well-developed network of stations, for the earthquakes in Southern Sumatra (4 June 2000) and off the coast of Central America (13 January 2001), it was impossible to select arrays meeting the applicability conditions of the method for determining the SAW wave vector parameters, as described in Sect. 3.1.

It is evident from the geometry in Fig. 1b,c that the earthquake epicenters lay inside the GPS arrays, which does not meet the far-field zone condition. It is possible that this is also responsible for the form of the response (presence of strong oscillations), which differs from the $N$-form of the response for the earthquakes in Turkey. On this basis, for these earthquakes, we can only point out the very fact of reliable detection of the response, and determine its amplitude $A_{I}$, typical period $T$, delay $\Delta t=t_{p}-t_{0}$, and velocity $V_{a}$ (see Table 3 ). The values of these quantities were close to the data obtained for the earthquakes in Turkey.

\section{Discussion}

The data in Table 3 are quite sufficient to estimate the position of the disturbances under discussion in the diagnostic diagram of the atmospheric waves. Specifically, the values of the characteristic periods of bipolar signals, presented in Fig. $2 \mathrm{~b}$,e, are $T_{1} \approx 300 \mathrm{~s}$, and $T_{2} \approx 200 \mathrm{~s}$. The wave vectors are at the angles $\beta_{1} \approx 70^{\circ}$ and $\beta_{2} \approx 50^{\circ}$ with respect to the vertical. At the height $z \approx 400 \mathrm{~km}$, in turn, the periods, corresponding to local frequencies of the acoustic cutoff $\omega_{a}$ and the Brunt-Väisälä $\omega_{b}$, are: $T_{a}=2 \pi / \omega_{a} \approx 950 \mathrm{~s}$, $T_{b}=2 \pi / \omega_{b} \approx 1050 \mathrm{~s}$.

In an isothermal atmosphere, only harmonics with periods larger than $T_{b} / \sin \beta$ can be assigned to the branch of internal gravity waves. This value significantly exceeds the values of $T_{1,2}$. Furthermore, $T_{1,2}$ is considerably smaller than $T_{a}$. Hence, we can contend that under conditions of the real (nonisothermal) atmosphere, the disturbances under discussion pertain almost entirely to the branch of acoustic waves.

Since in (short period) acoustic waves, the variation $\Delta N$ in neutral density $N$ satisfies the relation $\Delta N / N \approx u / C(u$ is the gas velocity in the wave, and $C$ is the local velocity of sound), it is possible to make a lower estimate of the intensity $u / C$ of the waves at the expected altitudes $z_{\text {eff }} \approx 350-$ $400 \mathrm{~km}: \quad \Delta N / N \approx \Delta N_{e} / N_{e} \approx \Delta I / I \approx 0.02-0.04$. In this estimation, the influence of the magnetic field is omitted, and the index $e$ refers to electrons. The actual intensity of the acoustic wave must be higher than the estimated value presented above. 
In the case of an earthquake, the movements of the terrestrial surface are plausible sources of acoustic waves. A generally known source of the first type is the Rayleigh surface wave, propagating from the epicentral zone. The ground motion in the epicentral area is the source of the second type. Let us discuss these possibilities.

\subsection{The Rayleigh wave}

The phase propagation velocity of the Rayleigh wave $V_{R} \approx$ $3.3 \mathrm{~km} / \mathrm{s}$. Since $V_{R} \gg C_{0}$, where $C_{0} \approx 0.34 \mathrm{~km} / \mathrm{s}$ is the sound velocity at the ground, only acoustic waves can be emitted (Golitsyn and Klyatskin, 1967). At a sufficient distance from the epicenter, where the curvature of the Rayleigh wave front can be neglected, and with the proviso that $\omega \gg$ $\omega_{a}$, acoustic waves are emitted at the angle $\beta_{R} \approx \arcsin \left(C_{0} /\right.$ $\left.V_{R}\right) \approx 6^{\circ}$ with respect to the vertical.

Rayleigh waves propagate generally in the form of a train consisting of several oscillations whose typical period rarely exceeds several tens of seconds. Acoustic waves are emitted upward in the form of the same train. Due to a strong absorption of the periodic wave, the only thing that is left over in the case of the acoustic train at heights $z \geq 350 \mathrm{~km}$ is the leading phase of compression. It seems likely that only in the case of strong earthquakes (Alaskian earthquake of 1964), even at large distances from the epicenter, the disturbance (the leading portion of the acoustic train) that remains from the acoustic train, can have at these altitudes a duration of about 100 $\mathrm{s}$, and quite an appreciable $(u / C>0.1)$ intensity (Orlov and Uralov, 1987), the nonlinear acoustics approximation.

Unfortunately, the parameters of Rayleigh waves in the neighborhood of the subionospheric points appearing in Table 3 are unknown to us. However, a most pronounced bipolar character of the main signal (Figs. 2b,e, 3b,e), its intensity, and its long duration cast some doubt upon the fact that it is the Rayleigh wave which is responsible for its origin. Such a conclusion is consistent with the observed propagation direction of the bipolar pulse, which makes a large angle with respect to the vertical: $\beta_{1,2} \approx 70-50^{\circ} \gg \beta_{R}^{\prime \prime} \approx 15-18^{\circ}$.

Here it is taken into consideration that the acoustic ray originating from a point on the ground at the angle of $\beta_{R} \approx$ $6^{\circ}$ now forms at the height $z=z_{\text {eff }}$ an angle $\beta_{R}^{\prime \prime}>\beta_{R}$ because of the refraction effect in a standard atmospheric model: $C_{0} / \sin \beta_{R}=C / \sin \beta_{R}^{\prime \prime}=$ const $=V_{R}, C(z=$ $\left.z_{\text {eff }}\right) \approx 0.9-1 \mathrm{~km} / \mathrm{s}$.

The presence of strong winds at ionospheric heights can alter the value of $\beta_{R}$. However, irrespective of the atmospheric model, the phase velocity $V_{h}$ (Table 3 ) of the horizontal trace of the acoustic disturbance generated by the Rayleigh wave must coincide with $V_{R}$, and this is also not observed: $V_{h} \ll V_{R}$.

\subsection{The epicentral emitter}

The detection of ionospheric disturbances, which are presumably generated by a vertical displacement of the terrestrial surface directly in the epicentral zone of an earthquake, using the GPS probing method, is reported in Calais and Minster (1995).

Results of the present study lend support to the above conjecture. However, the specific formation mechanism for the disturbance itself is still unclear. An approach to solving this problem is contained in earlier work, and involves substituting the epicentral emitter for a surface velocity point source or an explosion. In particular, the substitution of the earthquake zone for a point source turns out to be fruitful when describing long-period internal gravity waves at a very long (thousands of kilometers) distance from the epicenter (Row, 1967). The visual resemblance of ionospheric disturbances at short (hundreds of kilometers) distances from the earthquake epicenter to disturbances from surface explosions is discussed in Calais et al. (1998).

It should be noted that ionospheric disturbances generated by industrial surface and underground nuclear explosions are also visually similar. However, the generation mechanisms for disturbances are fundamentally different in this case $(\mathrm{Ru}-$ denko and Uralov, 1995). The radiation source in underground nuclear tests is, as in the case of earthquakes, the terrestrial surface disturbed by the explosion. The intensity and spectral composition of the generated acoustic signal reveal a strong (unlike the surface explosion) dependence on the zenith angle, and are wholly determined by the form, the size, and the characteristics of the movement of the terrestrial surface in the epicentral zone of the underground explosion.

In this section, we shall propose a model which, we hope, will help to understand the generation mechanism for acoustic disturbances: the subject of this paper. Because of the complexity of the problem, and the lack of sufficient data on characteristics of the movement of the terrestrial surface in epicentral zones of earthquakes, the idealized model under discussion has an illustrative character. The computational scheme proposed below represents a simplified variant of the scheme used in Rudenko and Uralov (1995) to calculate ionospheric disturbances generated by an underground confined nuclear explosion.

\subsection{The problem of radiation of the acoustic signal}

For the sake of simplicity, we consider a problem having an axial symmetry about the vertical axis $z$ passing through the earthquake epicenter $r=z=0$. The epicentral emitter is a set of plane annular velocity sources with the specified law of motion along the vertical $U(r, t)$. Since our interest is with the estimation of the characteristics of an acoustic disturbance at a sufficient distance from the emitter, we take advantage of the far-field approximation of a linear problem of radiation. In the approximation of linear acoustics $\omega \gg$ $\omega_{a}$ and with no absorption present, the gas velocity profile in the wave can be estimated by the expression:

$$
u(\tau, \beta)=\frac{A}{\pi R C_{0}} \int_{0}^{L} \int_{-\infty}^{+\infty} \frac{a\left(t^{\prime}, r\right) r d r d t^{\prime}}{\left.\sqrt{y^{2}-\left(\tau-t^{\prime}\right)^{2}}\right|_{\left|\tau-t^{\prime}\right| \leq y}}
$$



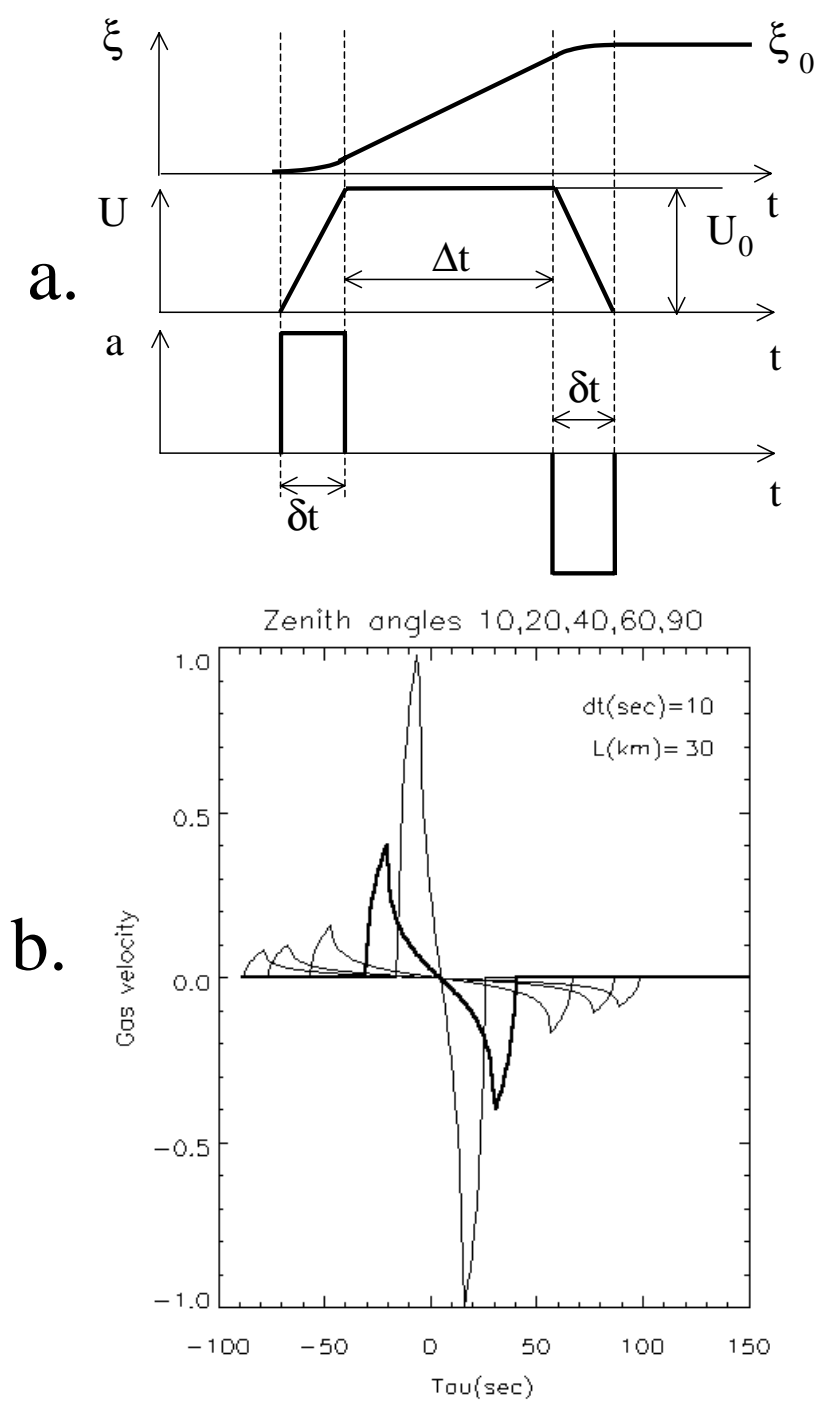

Fig. 4. (a) Model time dependence (from top to bottom) of the vertical displacement $\xi$, the velocity $U=d \xi / d t$ and the acceleration $a=d U / d t$ of the terrestrial surface in the epicentral zone of the earthquake. (b) Acoustic signals $u(\tau, \beta)$ in the far-field radiation zone of the piston $2 L=60 \mathrm{~km}$ in diameter. The piston's velocity has the form of a rectangular impulse of a duration $\Delta t=10 \mathrm{~s}$. The signals correspond to the expression (12) under the assumption of a homogeneous atmosphere; $A=1$, and $C=C_{0} \approx 0.34 \mathrm{~km} / \mathrm{s}$. The zenith angles are $\beta=10^{\circ}, 20^{\circ}, 40^{\circ}, 60^{\circ}$, and $90^{\circ}$. The abscissa axis indicates the time $\tau$ in seconds, and the axis of ordinates indicates the gas velocity $u$. The amplitude of the strongest signal $\beta=10^{\circ}$ is taken to be unity.

where $\tau=t-\int_{0}^{l} d l / C, \quad y=r \sin \beta / C_{0}$. Here, $\beta$ is the zenith angle of departure of the acoustic ray from the point $r=z=0 ; l$ is the group path length (the distance along the ray); $a\left(t^{\prime}, r\right)=d U(r, t) / d t$ is the vertical acceleration of the terrestrial surface; $A=A(z)=\sqrt{C_{0} \rho_{0} / C \rho}$ is the acoustic factor; $\rho_{0}, \rho$ stand for the air density at the ground and at the height $z$, respectively, and $\mathrm{L}$ is a typical radius of the epicentral emitter.
In an isothermal atmosphere where the ray trajectories are straight lines, the quantity $R=\left(\sqrt{r^{2}+z^{2}}=l\right)$ is the radius of a divergent (in the far-field approximation) spherical wave. In this case, the cross section of the selected ray tube $S \propto R^{2}$. In the real atmosphere, the value of $S$ is determined from geometrical optics equations, and using the expression (10), requires a further complication of the computational scheme. Nevertheless, to make estimates, we shall use only the relation (10), and, in doing so, the value of $R$ will be corrected.

The case $\beta=0$ (and also $l=R=z$ ) is a special one:

$u(\tau, \beta=0)=\frac{A}{R C_{0}} \int_{0}^{L} a(\tau, r) r d r$

Let us discuss the situation where all ring-type emitters 'operate' synchronously: $U(r, t)=U(t)$. In this case, the epicentral zone is emitting as a round piston $2 L$ in diameter. Assume also that with a shock of the earthquake, the vertical displacement $\xi$, the velocity $U=d \xi / d t$ and the acceleration $a=d U / d t$ are time dependent, as shown in Fig. 4a. For a rectangular velocity impulse, i.e. in the limit $\Delta t \gg \delta t \rightarrow 0$ (in this case, $a \delta t \rightarrow \pm U_{0} \delta\left(t^{\prime}\right)$, where $\delta\left(t^{\prime}\right)$ is a delta-function), from (10) we can obtain

$$
\begin{aligned}
u(\tau, \beta)= & \frac{U_{0} C_{0} A}{\pi R \sin ^{2} \beta}\left\{\left.\sqrt{y_{L}^{2}-\tau^{2}}\right|_{|\tau| \leq y_{L}}\right. \\
& \left.-\left.\sqrt{y_{L}^{2}-(\tau-\Delta t)^{2}}\right|_{|\tau-\Delta t| \leq y_{L}}\right\}
\end{aligned}
$$

where $y_{L}=L \sin \beta / C_{0} ; U_{0}, \Delta t$ are the amplitude and duration of the rectangular velocity impulse. In this case, the vertical displacement of the terrestrial surface after the earthquake shock is $\xi_{0}=U_{0} \Delta t$. In the strict sense, the expression (12) holds true if the condition $y_{L} \gg \delta t$ is satisfied, i.e. with zenith angles $\beta \gg \beta^{*} \approx \arcsin C_{0} \delta t / L$. Here $\delta t$ is the operation duration of the terrestrial shock at the beginning of the movement and at the stop of the piston. When $\beta \approx \beta^{*} \ll 1$, the expression (12) and the expression (11), in view of $\delta t \neq 0$, yield approximately identical signals. What actually happens is that the validity of the expression (12) will break down even earlier, and we are justified in using it only for zenith angles $\beta>\beta^{* *}=\arctan (L / z)>\beta^{*}$.

The curves in Fig. $4 \mathrm{~b}$ give an idea of the relative amplitude and form of acoustic signals $u(\tau, \beta)$ in the far-field zone of radiation of the piston $2 L=60 \mathrm{~km}$ in diameter. The duration of the rectangular velocity impulse of the piston was chosen arbitrarily, $\Delta t=10 \mathrm{~s}$. The signals correspond to the expression (12) under the assumption of a homogeneous, $A=1, C=C_{0} \approx 0.34 \mathrm{~km} / \mathrm{s}$, atmosphere. The zenith angles are $\beta=10^{\circ}, 20^{\circ}, 40^{\circ}, 60^{\circ}, 90^{\circ}$. The spherical surface $R=$ const serves as a reference. The abscissa axis indicates the time $\tau$ in seconds, and the axis of ordinates indicates the gas velocity $u$. In this case, the amplitude of the most intense signal $\left(\beta=10^{\circ}\right)$ is taken to be unity.

With increasing $\beta$, the amplitude of the signals $u_{\max }$ decreases, and the duration $T$ increases. The leading and trailing edges of the bipolar pulses in Figs. 2 and 3 have an equal 
duration $\Delta t$. The positive part of the bipolar pulse corresponds to the compression phase of the acoustic wave, and the negative part refers to the rarefaction phase. The area of the compression phase (in coordinates $u, t$ ) equals the largest displacement $\chi_{+}$of a unit volume of the atmosphere in the direction of propagation (along the ray) of the wave. A total area of a bipolar pulse is zero: $\chi_{+}=-\chi_{-}$. For acoustic signals, described by the expression (12), the following useful relations hold true:

$u_{\max }=U_{0} \frac{2 A L}{\pi R \sin \beta} \sqrt{\eta-\eta^{2}} ; \quad T=2 y_{L}+\Delta t$

$\chi_{+}=\xi_{0} \frac{A L}{2 \pi R \sin \beta}\left\{\sqrt{1-\eta^{2}}+\frac{1}{\eta} \arcsin \eta\right\} ; \quad \eta=\frac{\Delta t}{2 y_{L}}$

\subsection{The problem of acoustic signal propagation}

The wave vectors $\boldsymbol{K}_{t}$ of the bipolar pulses form, with respect to the vertical, the angles $\beta_{1}=70^{\circ}$ and $\beta_{2}=50^{\circ}$. With the adopted values of $z_{\text {eff }}=350-400 \mathrm{~km}$, the distances of the corresponding subionospheric points from the earthquake epicenters in Fig. 1a are approximately $r_{1}=800 \mathrm{~km}$ and $r_{2}=600 \mathrm{~km}$. The rays, constructed in the approximation of linear geometrical acoustics (LGA) and having at the heights $z=z_{\text {eff }}\left(C\left(z=z_{\text {eff }}\right) \approx 0.9-1 \mathrm{~km} / \mathrm{s}\right)$, the propagation angles $\beta_{1}$ and $\beta_{2}$ correspond to the zenith angle of departure $\beta \approx$ $19-21^{\circ}$ and $\beta \approx 15-17^{\circ}$, at the level $z=0$.

The fact that these values of $\beta$ satisfy the inequality $\beta \leq$ $\beta^{*} \approx 25-30^{\circ}$, is in reasonably good agreement with the familiar picture of rays from a ground-level point source; the rays with $\beta \geq \beta^{*}$ are captured by the atmospheric waveguide $z \leq z^{*}=120 \mathrm{~km}$, and only the rays emitted upward inside the solid angle $\Omega^{*} \approx 1$ sterad can penetrate to the heights $z>z^{*}$. In standard models of the atmosphere, however, for the values of the angles $\beta_{1,2}\left(z=z_{\text {eff }}\right)$, there are corresponding locations of subionospheric points lying several hundreds of kilometers closer to the epicenter, compared to the experimental values of $r_{1} \approx 800 \mathrm{~km}$ and $r_{2} \approx 600 \mathrm{~km}$. This inconsistency can be caused by two reasons. One reason is that the value of $z_{\mathrm{eff}} \approx 350-400 \mathrm{~km}$, which we are using, is too low. This is supported by the detection of velocities $V_{t} \approx 1.2 \mathrm{~km} / \mathrm{s}$ of traveling disturbances (Table 3 ), which markedly exceed the sound velocity $C \approx 0.9-1 \mathrm{~km} / \mathrm{s}$, at the heights of $\approx 350$ $400 \mathrm{~km}$. However, it seems likely that such a discrepancy may be disregarded, in view of the errors in the measurement technique used (the probability of an additional heating of the upper atmosphere prior to the earthquake cannot be ruled out, however). The increase of the actual value of $z_{\text {eff }}$ can also be associated with a strong dependence (see (13), (14) and Fig. 4b) of the power of the emitted signal on the zenith angle of departure of the ray from the earthquake epicenter. Verifying this factor requires a more detailed analysis based on particular data on vertical movements of the terrestrial surface in the epicentral zone; such data are unavailable to us. We devote our attention now to the second reason for the above-mentioned inconsistency, and consider it to be highly probable. The second reason may be associated with the violation of the validity conditions of the LGA approximation at a sufficient distance from the source of the acoustic disturbances under discussion. Indeed, the utilization of this approximation is justified until the parameters of the medium and of the wave itself change substantially, based on the size of the first Fresnel zone $d_{F} \approx \sqrt{\lambda l}$, which determines the physical (transverse) size of the ray. Typical wavelengths of the bipolar pulses under discussion at the heights $z=z_{\text {eff }}$ are large: $\lambda \approx 300-200 \mathrm{~km}$. Distance $l$ along the expected ray is of the order of 900-700 km. Then $d_{F} \approx 520-370 \mathrm{~km}$, which substantially exceeds the scales of variation of atmospheric parameters. The value of $d_{F}$ is actually somewhat smaller, because the typical scale of a disturbance decreases as it approaches the source. A violation of the LGA approximation at the above-mentioned distances from the epicentral source also occurs for model signals $\beta \geq 20^{\circ}$, as shown in Fig. 4b.

The increasing violation of the applicability conditions of the LGA approximation with an increase of $l$ implies the transport of the wave energy not strictly along the calculated rays, but also along the lines with a smaller curvature. With a mere estimate of the dilution factor $R$ in the expressions (12), (13) and (14), these lines are assumed to be straight when $z>z^{*}$ and originate from an imaginary source lying at the height $z^{*} \approx 120 \mathrm{~km}$ above the earthquake epicenter. Such a situation is also clearly manifested in the LGA approximation. At the heights $z>z^{*}$, at $t \approx 600 \mathrm{~s}$, for example, the surface of the wave front from the groundlevel impulsive source resembles the surface of a hemisphere centered on the point $r \approx 0, z \approx z^{*}$.

Ultimately the energy that arrives from below, inside the solid angle $\Omega^{*} \approx 1$ is scattered into a solid angle $\Omega \approx 2 \pi$. From the condition of conservation of wave energy, it is possible to find $R_{1,2}=\sqrt{\Omega / \Omega^{*}} \sqrt{(\Delta z)^{2}+r_{1,2}^{2}}$, when $\Delta z=$ $z_{\text {eff }}-z^{*}$. When using the expressions (13), (14) in the subsequent discussion, we will take the quantity $R_{1}$ rather than $R$, and the value of the zenith angle of departure will be taken to be $\beta=20^{\circ}$. These parameters approximately correspond to the generation and propagation conditions of the signal from the first earthquake. The typical size of the epicentral zone of this earthquake was about $2 L=60 \mathrm{~km}$ (according to the USGS data: www.neic.cr.usgs.gov). This same value of $L$ was used in calculating the signals shown in Fig. 4b. As is evident even from Fig. $4 b$ (thick line), the duration of the signal, having a zenith angle of departure $\beta=20^{\circ}$, is about $70 \mathrm{~s}$. When the signal propagates in the approximation of linear acoustics and with no absorption, its form and duration remain unchanged, and only its amplitude changes.

In actual conditions, the combined effect of the nonlinear attenuation and linear absorption factors leads to a stretching of the bipolar pulse, and to a change of its form (we do not discuss the dispersion factor). In this case, the effect of the nonlinearly factor occurs in such a manner that the integral value of $\chi_{+},(14)$, calculated as an approximation of linear acoustics, remains as such in the approximation of nonlinear acoustic as well. Moreover, taking into account the finite width $\Delta T_{s h}$ of the shock front does not change this situa- 
tion until $\Delta T_{s h}<T / 4$. The linear absorption factor, in turn, somewhat reduces the true value of $\chi_{+}$because of the mutual diffusion of the compression and rarefaction phases. Nevertheless, for a hypothetical estimation of the earthquake parameters, we shall use the assumption about the conservation of the value of $\chi_{+}$.

As is intimated by Table 3, the mean value of the $T E C$ disturbance amplitude after the first earthquake is $A_{I} \approx 0.14$ TECU at the equilibrium value of $I \approx 5$ TECU. Assuming that on the order of magnitude of $A_{I} / I \approx u / C$ for a maximum gas velocity in the wave, we have an estimate of $u^{\exp } \approx 30 \mathrm{~m} / \mathrm{s}$. For the sinusoidal form of the bipolar pulse with a duration $T^{\exp } \approx 350 \mathrm{~s}$, we find the experimental value of gas displacement along the direction of wave propagation: $\chi_{+}{ }^{\exp }=u^{\exp } T^{\exp } / \pi \approx 3 \mathrm{~km}$. Using the relation $\chi_{+}{ }^{\exp } \approx \chi_{+}$, it is possible to estimate the vertical displacement $\xi_{0}$ of the terrestrial surface in the epicentral zone of the earthquake. For this purpose, it seems reasonable to introduce the assumption about a short velocity impulse which models the main earthquake shock, $\eta \ll 1$, although the numerical value of $\chi_{+}$is virtually independent of the value of $\eta$ (14). In view of the above considerations, we then obtain:

$\xi_{0} \approx \chi_{+}{ }^{\exp } \pi R_{1} \sin \beta /(A L)$.

The uncertainty in the determination of $\xi_{0}$ is caused both by the uncertainty of the true values of the quantities involved in this relation and by the limitations of the acoustic signal generation model itself. In this case, of the greatest importance is the dependence of $\xi_{0}$ on the value of the acoustic parameter $A$, containing the atmospheric density $\rho$ at the effective height $z=z_{\text {eff }}$, which we have introduced artificially. The employment of the MSISE90 atmospheric model (Hedin, 1991), calculated for the location and time of the first earthquake, gives the values of $\xi_{0} \approx 60,40$ and $25 \mathrm{~cm}$, with the values of $z_{\mathrm{eff}}=350,400$ and $450 \mathrm{~km}$, respectively. In all cases, it was assumed that $R_{1} \approx 850 \sqrt{2 \pi} \mathrm{km}, \beta=20^{\circ}$, $L=30 \mathrm{~km}$. The possibility of a vertical displacement of the terrestrial surface in the epicentral zone by several tens of centimeters seems real. In particular, Calais and Minster (1995) give the value of $\xi_{0} \approx 40 \mathrm{~cm}$ at the epicenter of the $M w=6.7$ Northridge earthquake (California, 1994).

In view of the demonstration character of the above calculation, we have intentionally excluded from consideration the effects associated with the inclination of the magnetic field lines, and with the possible presence of strong winds at ionospheric heights. The presence of a magnetic field modifies the picture concerning the transfer of movements from the neutral gas to the electron component of the ionosphere. Since the magnetic field is not entrained by the neutral gas, the field lines can be considered fixed. In this case, the acceptable approximation would be the one in which the electron component travels only along magnetic field lines with the velocity $u \cos \psi$, where $\psi$ is the angle between the magnetic field vector and the velocity vector of the neutral gas. Therefore, the quantity $u \cos \psi$ must be involved in lieu of the quantity $u$ in the expression $A_{I} / I \approx u / C$ that was used above.
Let us estimate the value of $\psi$ for Turkey's earthquakes. In the examples under discussion (Table 3), the horizontal projection $\boldsymbol{K}$ of the full wave vector $\boldsymbol{K}_{t}$ is virtually collinear to the horizontal component of the magnetic field. The wave vectors $\boldsymbol{K}_{t}$, in turn, form angles from $\beta_{1} \approx 70^{\circ}$ to $\beta_{2} \approx 50^{\circ}$ with respect to the vertical.

Since the magnetic dip in the middle of Turkey is about $60^{\circ}$ (the angle is measured from the horizontal plane), $\psi \cong$ $20^{\circ}-40^{\circ}$, and the value of $\cos \psi \approx 0.94-0.77$ hardly differs from 1. It should be remembered, however, that taking into account this factor can be very important in the analysis of the complete picture of TEC disturbances above the earthquake or explosion source (see, for example, Calais et al., 1998).

The presence of the zonal and meridional winds at ionospheric heights leads to a displacement and deformation of the wave front, and hence, gives rise to a dependence of the acoustic wave intensity on the propagation direction. The decisive role in this case is played by the wind velocity gradient. This factor can be taken into account within the framework of the ray theory. However, a corresponding model calculation would be worthwhile in the analysis of experimental data obtained for a set of subionospheric points surrounding the acoustic wave source. In the present situation, however, where the number of subionospheric points used in the analysis is too small, and the uncertainty of the parameters of the acoustic emitter itself is too large, the solution of such an unwieldy problem would be an overrun of the accuracy which is pursued by the above computational scheme.

As follows from the expressions (13), maximum values of displacements and the velocity of the neutral atmospheric species are attained directly above the earthquake epicenter. The signal duration is minimal, and does not seem to exceed a few tens of seconds at ionospheric heights. Since the wave vector of the disturbance is directed predominantly upward, the method of oblique-incidence ionospheric sounding, in this case, is the technique of choice for determining the waveform.

\section{Conclusion}

In this paper we have investigated the form and dynamics of shock-acoustic waves generated during earthquakes. We have developed a method of determining the SAW parameters using GPS arrays whose elements can be chosen out of a large set of the global network GPS stations. Unlike existing radio techniques, the proposed method estimates the SAW parameters without a priori information about the location and time of the earthquake. The implementation of the method is illustrated by an analysis of ionospheric effects of the earthquakes in Turkey (17 August and 12 November 1999), in Southern Sumatra (4 June 2000), and off the coast of Central America (13 January 2001).

It was found that, in spite of the difference of the earthquake characteristics, the local time, the season, and the level of geomagnetic disturbance, for the four earthquakes, the 
time period of the ionospheric response is $180-390 \mathrm{~s}$, and the amplitude exceeds, by a factor of two as a minimum, the standard deviation of background fluctuations in total electron content in this range of periods under quiet and moderate geomagnetic conditions.

As has been pointed out in the Introduction, some investigators report markedly different values of the SAW propagation velocity, by as much as several thousands $\mathrm{m} / \mathrm{s}$, which is beyond the values of the sound velocity of the SAW propagation heights in the atmosphere. The method proposed in this paper opens up a possibility of determining the angular characteristics of the wave vector $\boldsymbol{K}_{t}$ and, accordingly, of estimating $V_{t}$. According to our data (Table 3), the elevation of the SAW wave vector varied within $20-44^{\circ}$, and the phase velocity of the SAW varied from 1100 to $1300 \mathrm{~m} / \mathrm{s}$. We determine the phase velocity of the equal TEC line at the height of the ionospheric F-region maximum, which makes the main contribution to variations of the TEC between the receiver and the GPS satellite, and corresponds to the region of maximum sensitivity of the method. Since $V_{t}$ approaches the sound velocity at these heights ( $\mathrm{Li}$ et al., 1994), this makes it possible to identify the sound origin of the TEC disturbance. The SAW source location, calculated without taking into account the refraction corrections, approximately corresponds to the earthquake epicenter.

Acknowledgements. We are indebted to G. M. Kuznetsova and A. V. Tashchilin for their calculation of the atmospheric parameters at the time of the earthquakes, as well as to E. A. Ponomarev, V. V. Evstafiev, P. M. Nagorsky, N. N. Klimov, and A. D. Kalikhman for their interest in this study, many pieces of useful advice, and active participation in discussions. Thanks are also due to V. G. Mikhalkosky for his assistance in preparing the English version of the manuscript. Finally, the authors wish to thank the referees for valuable suggestions which greatly improved the presentation of this paper. This work was done with support from both the Russian foundation for Basic Research (grant 99-05-64753) and RFBR grant of leading scientific schools of the Russian Federation No. 00-15-98509.

Topical Editor M. Lester thanks K. Davies and E. Calais for their help in evaluating this paper.

\section{References}

Afraimovich, E. L., Varshavsky, I. I., Vugmeister, B. O., et al., Influence of surface industrial explosions on Doppler and angular characteristics of the ionosphere-reflected radio signal, Geomagnetizm i aeronomiya, 24, 322-324, 1984.

Afraimovich, E. L., Terechov, A. I., Udodov, M. Yu., and Fridman, S. V., Refraction distortions of transionospheric radio signals caused by changes in a regular ionosphere and by travelling ionospheric disturbances, J. Atmos. Terr. Phys., 54, 1013-1020,
1992.

Afraimovich, E. L., Palamartchouk, K. S., and Perevalova, N. P., GPS radio interferometry of travelling ionospheric disturbances, J. Atmos. Terr. Phys., 60, 1205-1223, 1998.

Blanc, E. and Jacobson, A. R., Observation of ionospheric disturbances following a 5-kt chemical explosion. 2. Prolonged anomalies and stratifications in the lower thermosphere after shock passage, Radio Sci., 24, 739-746, 1989.

Calais, E. and Minster, J. B., GPS detection of ionospheric perturbations following the January 1994, Northridge earthquake, Geoph. Res. Lett., 22, 1045-1048, 1995.

Calais, E. and Minster, J. B., GPS detection of ionospheric perturbations following a Space Shuttle ascent, Geoph. Res. Lett., 23, 1897-1900, 1996

Calais, E., Minster, J. B., Hofton, M. A., and Hedlin, M. A. H., Ionospheric signature of surface mine blasts from Global Positioning System measurements, Geoph. J. Int., 132, 191-202, 1998.

Davies, K., Ionospheric radio waves, Blaisdell Publishing Company, Waltham, Massachusetts-Toronto-London, 1969.

Fitzgerald, T. J., Observations of total electron content perturbations on GPS signals caused by a ground level explosion, J. Atmos. Terr. Phys., 59, 829-834, 1997.

Golitsyn G. S. and Klyatskin, V. I., Vibrations in the atmosphere caused by movements of the terrestrial surface, Izv. Akademii nauk SSSR, Fizika atmosfery I okeana, 111, 1044-1052, 1967.

Hedin, A. E., Extension of the MSIS thermosphere model into the middle and lower atmosphere, J. Geophys. Res., 96, 1152-1172, 1991.

Jacobson, A. R. and Carlos, R. C., Observations of acoustic-gravity waves in the thermosphere following Space Shuttle ascents, J. Atmos. Terr. Phys., 56, 525-528, 1994.

Li, Y. Q., Jacobson, A. R., Carlos, R. C., Massey, R. S., Taranenko, Y. N., and Wu, G., The blast wave of the Shuttle plume at ionospheric heights, Geoph. Res. Lett., 21, 2737-2740, 1994.

Mercier, C. and Jacobson, A. R., Observations of atmospheric gravity waves by radio interferometry: are results biased by the observational technique, Ann. Geophysicae, 15, 430-442, 1997.

Nagorsky, P. M., The inhomogeneous structure of the ionospheric F-region produced by rockets, Geomagnetizm i aeronomiya, 38 , 100-106, 1998.

Orlov, V. V. and Uralov, A. M., Atmospheric response to the Rayleigh wave generated by an earthquake, In: Issledovaniya po geomagnetismu, aeronomii i fizike Solntsa, Moscow: Nauka, 78, 28-40, 1987.

Pi, X., Mannucci, A. J., Lindgwister, U. J., and Ho, C. M., Monitoring of global ionospheric irregularities using the woldwide GPS network, Geophys. Res. Lett., 24, 2283-2286, 1997.

Row, R. V., Acoustic-gravity waves in the upper atmosphere due to a nuclear detonation and an earthquake, J. Geophys. Res., 72, 1599-1610, 1967

Rudenko, G. V. and Uralov, A. M., Calculation of ionospheric effects due to acoustic radiation from an undeground nuclear explosion, J. Atmos. Terr. Phys., 57, 225-236, 1995. 